Original Research Paper

\title{
Public Risk Perceptions of Handgun Use in the United States
}

\author{
Jenna Tyler and Abdul-Akeem Sadiq \\ School of Public and Environmental Affairs, Indiana University-Purdue University Indianapolis, \\ 801 West Michigan Street, Indianapolis, IN 46202, USA
}

Article history

Received: 12-04-2017

Revised: $12-06-2017$

Accepted: 14-07-2017

Corresponding Author: Jenna Tyler

School of Public and Environmental Affairs, Indiana University-Purdue University Indianapolis, 801 West Michigan Street, Indianapolis, IN 46202, USA Email: jentyler@iupui.edu

\begin{abstract}
This study explores public risk perceptions of other people's use of handguns and the influence of demographics on individuals' risk perceptions of other people's use of handguns. Data were collected from a nationally representative online survey of 2,008 individuals in the United States. The results indicate that the public views other people's use of handguns as highly risky (3.6 on a scale of 1 to 5). This result is not particularly interesting by itself, but when analyzing individuals' risk perceptions of other people's use of handguns along various demographics, interesting insights emerge. Specifically, the results indicate that women and minority groups, as well as older and more educated individuals have a significantly higher risk perception of other people's use of handguns. A better understanding of public risk perceptions of other people's use of handguns can provide critical insights needed to design appropriate gun safety policies.
\end{abstract}

Keywords: Risk Perception, Handguns, Gun Control

\section{Introduction}

Despite remarkable decreases in firearm related deaths in the United States since the mid-1990s (Cohn et al., 2013), gun control remains one of the most polarizing policy issues. Notable mass shootings such as the Columbine High School shooting, the Aurora, Colorado movie theater shooting, the Sandy Hook Elementary School shooting and more recently, the Orlando nightclub shooting have placed this issue at the top of political agendas. Advocates for more stringent firearm legislation suggest that banning military-style assault weapons and implementing more rigorous background checks will reduce the annual number of firearm-related deaths and injuries (Celinska, 2007; Stroebe, 2015). Conversely, opponents of stricter gun laws argue that such efforts infringe upon their second amendment right and will do little to reduce the actual number of firearm-related fatalities (Celinska, 2007). These individuals further suggest that firearms are used for self-defense and limiting access to firearms for law-abiding citizens will endanger public safety (Celinska, 2007; Stroebe, 2015).

In response to these conflicting views, scholars have extensively investigated various aspects of gun violence. For example, scholars have empirically investigated the relationship between gun sale laws and gun availability
(Webster et al., 2001), gun ownership and social gun culture (Kalesan et al., 2016), gun purchase and mortality from firearm injury (Grassel et al., 2003) and gun availability and gun violence (Konty and Schaefer, 2012). In addition, scholars have examined individuals' perceptions of gun policies (Teret et al., 1998), as well as the relative incidence of gun victimization versus selfdefense gun use by individuals in the United States (Hemenway et al., 2000). Although these studies and others have significantly contributed to the gun control debate, they neglect to provide a thorough understanding of how individuals perceive the risk associated with using firearms. This is surprising given that scholars suggest that "the gun control debate is naturally framed as one involving competing perceptions of risk" (Kahan and Braman, 2003: 1299). Additionally, despite an extensive collection of research on public risk perceptions of hazardous activities and technologies (Cutter et al., 1992; Fischhoff et al., 1978; Slovic et al., 1979), the relationship between public risk perceptions of other people using handguns and demographic characteristics has yet to be studied. This is also surprising given that, aside from gun ownership, demographic characteristics remain the strongest predictors of attitudes toward gun control (Kahan and Braman, 2003). The purpose of this study is thus to address these gaps in literature. 
This study contributes to the risk perception and guncontrol literature by addressing the following two research questions. (1) What is the public's risk perception of other people's use of handguns? (2) What is the relationship between demographic characteristics and risk perceptions of other people's use of handguns? Answers to these questions will provide important insights to policymakers and public safety practitioners in charge of developing policies and programs related to firearms. For example, an understanding of how the American public views the risk of another person using a handgun will aid policymakers and practitioners in their efforts to educate the public on the risks associated with using firearms, thus improving risk communication and ultimately, gun safety. Moreover, from a theoretical standpoint, this study provides clarity on the direction and significance of the relationship between demographic characteristics (e.g., age, gender, income, educational level, etc.) and public risk perceptions of other people's use of handguns. In addition, our study answers Kahan and Braman's (2003) call for scholars to contribute to the gun control debate by studying public risk perceptions, as well as Rader et al. (2007) call for more research on the relationship between perceived risk and demographic variables.

The remainder of this paper is organized into four sections. The first section reviews the extant research findings on public risk perceptions of firearms. The second section outlines the data and methods used in this study. The third section presents the findings and includes a discussion of the results. The final section discusses study limitations and outlines an agenda for future research on public risk perceptions of handguns.

\section{Literature Review}

\section{Public Risk Perceptions of Firearms}

Scholars have primarily studied public risk perceptions of hazardous activities and technologies, including handguns, using two theories-the psychometric paradigm and the cultural theory of risk (Kahan et al., 2011). The psychometric paradigm involves asking respondents to quantitatively consider the risk of dying from various hazardous activities and technologies (Cutter et al., 1992; Fischhoff et al., 1978; Slovic et al., 1979). In addition, respondents provide judgments on other properties of the hazard such as the extent to which the hazard is dreadful, voluntary, new and controllable, as well as the benefits and consequences associated with the hazard (Fischhoff et al. 1978; Slovic et al. 1979). Arguably, the most important aspect of the psychometric paradigm is the assumption that risk is inherently subjective, implying that an individual's risk perception remains bounded by their psychological and decisionmaking processes (Sjöberg et al., 2004).
Scholars have employed the psychometric paradigm to quantitatively determine what hazards individuals express extreme aversion to and what hazards individuals express an indifference to (Slovic et al., 1979). Slovic et al.'s (1979) seminal work, for example, employed the psychometric paradigm to explore public risk perceptions of handguns and 29 other hazardous activities and technologies such as smoking, motor vehicles and alcoholic beverages and then ranked them based on the size of the geometric mean risk values, starting with the highest value. Using survey responses from four different groups-League of Women Voters and their spouses, college students, active club members and experts- Slovic et al. (1979) found that the public perceives the risk of dying from a handgun to be relatively high. In fact, out of the 30 different hazardous activities and technologies, League of Women Voters and their spouses ranked the risk of dying from a handgun third; college students ranked the risk of dying from a handgun second; active club members ranked the risk of dying from a handgun first; and experts ranked the risk of dying from a handgun fourth (Slovic et al., 1979). Expanding on Slovic et al.'s (1979) work, Cutter et al. (1992) studied undergraduate students' risk perceptions of handguns and 32 other hazardous activities and technologies to determine the effect gender has on risk perceptions. The authors found, among other results, that both males and females ranked handguns as one of the top five riskiest hazardous activities and technologies (Cutter et al., 1992).

While the psychometric paradigm has provided valuable information on public risk perceptions of various hazardous activities and technologies relative to others, it fails to consider cultural factors that may influence individuals' risk perceptions. As a result, scholars have studied public risk perceptions using the cultural theory of risk (Kahan et al., 2011). The cultural theory of risk stems from the work of Douglas and Wildavsky (1983) and suggests that individuals form risk perceptions that reflect and sustain their way of life (Douglas and Wildvasky, 1983; Kahan et al., 2011; Song, 2014). In other words, the more an individual believes a hazard supports their way of life, the lower they will perceive the risk of the hazard (Song, 2014). Conversely, the more an individual believes a particular hazard threatens their way of life, the higher they will perceive the risk of the hazard (Song, 2014).

The cultural theory of risk provides a theoretical basis for explaining the nature and intensity of the American gun control debate as it considers the cultural factors that motivate individuals to support gun control policies (Kahan and Braman, 2003). Kahan and Braman (2003) explored public risk perceptions of gun control and found that individuals' perceptions of gun control stems from their cultural worldviews. Expanding on this work, Kahan et al. (2007) explored what cultural worldviews 
influence public risk perceptions of guns as well as abortions and other environmental hazards. The results of this study showed that egalitarian and communitarian worldviews predicted the belief that guns are dangerous while hierarchical and individualistic worldviews predicted the belief that guns are safe (Kahan et al., 2007). More specifically, Kahan et al. (2007) suggest that individuals with hierarchical-individualistic worldviews have lower risk perceptions of guns because they associate guns with more traditional gender and authoritative roles and believe guns are appropriate for individual self-reliance. Conversely, individuals with egalitarian-communitarian worldviews have higher risk perceptions of guns because they associate guns with gender and racial inequality and believe guns threaten the trust, solidarity and safety of communities (Kahan et al., 2007).

The purpose of this study is not to test the efficacy of the psychometric paradigm or the cultural theory of risk, but to use these theories as the basis for examining public risk perceptions of other people's use of handguns and exploring the relationship between public risk perceptions of other people's use of handguns and demographic characteristics. The studies employing the psychometric paradigm, for example, suggest that the American public perceives the risk of handguns to be relatively high (Cutter et al., 1992; Slovic et al., 1979). As a result, we posit that, in general, survey respondents will report a relatively high-risk perception of other people's use of handguns. Moreover, studies using the cultural theory of risk suggest that individual attitudes and worldviews influence public perceptions of the dangerousness of guns and can help explain socio-demographic differences in risk perceptions of guns (Kahan and Braman, 2003; Kahan et al., 2007). In the following section, we use the studies discussed above, as well as additional studies on public risk perceptions of hazardous activities and technologies, fear of crime and criminal victimization to form our hypotheses regarding the relationship between public risk perceptions of other people's use of handguns and the following demographic characteristics: Age, educational level, gender, ethnicity and household income.

\section{Age}

The relationship between age and risk perception is complex and inconclusive (Rader et al., 2007). For example, criminologists have found a positive (LaGrange and Ferraro, 1989), negative (Rountree and Land, 1996), or nonexistent (Hraba et al., 1998; Mesch, 2000) relationship between perceived risk of criminal victimization and age. Additional studies on public risk perceptions of natural disasters, automobile accidents, cancer and other risks also show that the relationship between age and risk perception is mixed. Some studies (e.g., Grothmann and Reusswig, 2006), for example, found a positive relationship between age and risk perception, suggesting that older individuals have higher risk perceptions. Yet, other studies (e.g., Savage, 1993; White et al., 2015) found a negative relationship between age and risk perception, implying that younger individuals have higher risk perceptions.

\section{Hypothesis 1: There will be a significant relationship between age and risk perception of other people's use of handguns.}

\section{Educational Level}

A large body of research on public risk perceptions of various hazardous activities and technologies (e.g., aviation accidents, natural disasters, etc.) suggests that there is a negative relationship between educational level and risk perception, implying that the less educated have higher risk perceptions (Dosman et al., 2001; Savage 1993). Moreover, Chiricos et al. (2000) found that those with lower levels of education have higher levels of perceived risk of criminal victimization. However, when specifically observing public risk perceptions of firearms, Kahan et al. (2007) found a positive and statistically significant relationship between education and perceived dangerousness of guns.

\section{Hypothesis 2: There will be a significant relationship between educational level and risk perception of other people's use of handguns.}

\section{Gender}

Studies examining the relationship between gender and risk perceptions of various hazardous activities and technologies, including the use of handguns, have repeatedly shown that women report higher risk perceptions than men (Barke et al., 1997; Gustafson, 1999; Kahan et al., 2007). For example, Kahan et al. (2007) found a positive and statistically significant relationship between females and perceptions that guns are dangerous. Moreover, when specifically examining perceptions of gun control, Kahan and Braman (2003) found a positive and statistically significant relationship between females and support for gun control. Despite directly experiencing gun violence at a lower rate than males (Planty and Truman, 2013), scholars suggest that because women are more attuned to the possibility of being a victim of a violent attack, they view handguns and other weapons as riskier than their male counterparts (Kahan and Braman, 2003).

\footnotetext{
Hypothesis 3: Females will have a significantly higher risk perception of other people's use of handguns than males.
} 


\section{Ethnicity}

Risk perception scholars have predominantly found that minority groups have a higher risk perception than whites (Kahan and Braman, 2003; Wilcox et al., 2003). For example, Kahan et al. (2007) found a positive and statistically significant relationship between blacks and perceived dangerousness of guns. Likewise, Finucane et al. (2000) concluded that racial minorities have significantly higher risk perceptions of criminal victimization as well as other hazardous activities and technologies (e.g., blood transfusions, natural disasters, etc.). These findings are not surprising given that minority groups, especially blacks and Hispanics, are more likely to be a victim of firearm violence than whites (Planty and Truman, 2013).

\section{Hypothesis 4: Minorities will have a significantly higher risk perception of other people's use of handguns than whites.}

\section{Household Income}

Similar to age and educational level, the literature on the relationship between household income and risk perception remains mixed. For example, Chiricos et al. (2000) found a negative relationship between household income and risk perceptions of criminal victimization while Hraba et al. (1998) found a positive relationship between income and individuals' perceived risk of criminal victimization. Kahan and Braman (2003) findings align with Hraba et al.'s (1998) conclusions, as these authors found a positive relationship between household income and perceptions that guns are dangerous.

\section{Hypothesis 5: There will be a significant relationship between household income and risk perception of other people's use of handguns.}

\section{Methods}

\section{Data Collection}

One of the authors was part of the research team that designed the survey instrument. After designing the survey instrument, it was pre-tested with 17 staff and alumni from a Midwestern university in the United States. This pre-test revealed a few minor problems that the research team corrected and the corrected survey was handed to GfK for administration. GfK, an established international survey company, sent the survey to individuals in their KnowledgePanel ${ }^{\circledR}$. The KnowledgePanel ${ }^{\circledR}$ consists of a nationally representative random sample of the United States population. Members of the KnowledgePanel ${ }^{\circledR}$ were recruited using random digit dialing and address-based sampling methods that include households with and without Internet access. If a household without access to the Internet or a computer was selected, GfK provided them with both at no charge. In short, the KnowledgePanel ${ }^{\circledR}$ provides a nearly complete coverage of the United States population.

GfK distributed the survey to a nationally representative sample of 10,599 adults in the United States, 18 years of age older, in their KnowledgePanel®. Of the 10,559 invited to participate, 5,079 responded. The respondents, however, had to meet two requirements set forth by the research team. First, respondents could not be self-employed. Second, respondents could not telecommute for the majority of their work time. Individuals who reported that they were self-employed or telecommuted for the majority of their work time were excluded from the study. This is because the main purpose of this survey was to understand employees' perceptions of their employer's level of disaster preparedness. Understanding current risk perceptions for hazardous technologies and activities, including the use of handguns, was a secondary purpose. These two requirements reduced the sample to 2,026 (2,702 were eliminated for being self-employed and 351 for telecommuting). An additional 18 surveys were removed from the analyses due to short completion times. Analyzing survey completion times is a common technique in survey research because it allows researchers to identify participants who may not have fully read each survey question or participants who exhibited 'straight-lining' (i.e., answering several items with the same response).

Based on the guidelines set forth by the AAPOR (2015), the study completion rate is $48 \%$. The completion rate was calculated by dividing the 2,008 interviews by the sum of 2,026 known eligible cases and 2,192 estimated eligible cases among the 5,480 who did not respond to the invitations (assuming an estimated eligibility rate of $40 \%$, based on the eligibility rate of the 5,079 respondents). To compute a cumulative responsive rate, this completion rate is multiplied by the KnowledgePanel ${ }^{\circledR}$ recruitment rate (those who agreed to join the panel) and profile rate (those who completed necessary profile surveys). According to GfK, the recruitment rate for this study was $13.9 \%$ and the profile rate was $65 \%$. This results in a cumulative response rate of $4.3 \%$. GfK weighted the data to account for unequal probabilities of selection as well as to ensure that the data were as close as possible to Current Population Survey (CPS) estimates for the United States population with respect to demographic characteristics.

\section{Variable Measurement}

The dependent variable for this study is risk perception of other people's use of handguns. This variable was measured by asking respondents the following survey question: "On a scale of 1 (not at all 
risky) to 5 (highly risky), please rate how risky you believe [using handguns (by others)] is in general". Asking individuals their perceived risk of another person using a handgun is appropriate because simply asking individuals their risk perception of a handgun may cause respondents to consider the weapon itself, not the intentions of the person who might use it (Boholm, 1998). Moreover, measuring public risk perceptions via Likert scales remains common across the risk perception literature (e.g., Fischhoff et al., 1978; Sadiq and Graham, 2015; Sadiq et al., in press).

The independent variables in this study include age, educational level, gender, ethnicity and household income. Age was measured using four age categories (18-29 years, 30-44 years, 45-59 years and 60+ years). Educational level was measured as less than high school $(=1)$, high school graduate/high school diploma $(=2)$, some college, no degree $(=3)$, associate degree $(=4)$, bachelor's degree $(=5)$, master's degree $(=6)$ and professional or doctorate degree $(=7)$. Gender was measured as a dichotomous variable (male $=1$, female $=$ $0)$. Ethnicity was measured by white, non-Hispanic (= $1)$, black, non-Hispanic $(=2)$, other, non-Hispanic $(=3)$, Hispanic $(=4)$ and $2+$ races, non-Hispanic $(=5)$. For the analyses, a new dichotomous variable was created (white $=1$ and black, non-Hispanic/Hispanic =0). Lastly, household income was operationalized as annual household income and was measured as less than $\$ 14,999(=1), \$ 15,000-\$ 29,999(=2), \$ 30,000-\$ 49,999$ (=3), \$50,000-\$74,999 (=4), \$75,000-\$99,999 (= 5) and over $\$ 100,000(=6)$.

Finally, based on the risk perception scholarship, this study employs four control variables: Household size, marital status, ownership of living quarters and parental status (presence of child/children). Household size was measured as the number of individuals in a household: 1 $(=1), 2(=2), 3(=3), 4(=4), 5$ or more $(=5)$. Marital status was measured as married $(=1)$, widowed $(=2)$, divorced $(=3)$, separated $(=4)$, never married $(=5)$ and living with partner $(=6)$. Ownership status of living quarters was measured as owned or being bought by you or someone $(=1)$, rented for cash $(=2)$, occupied without payment of cash rent $(=3)$. Lastly, parental status was coded 1 for respondents with at least one child and 0 for those with no child.

\section{Results}

Table 1 displays the demographic characteristics of the 2,008 respondents. Approximately 37\% of respondents are between the ages of 45 and 59. In terms of education, $26.1 \%$ have a bachelor's degree and $12.9 \%$ have a master's degree. Furthermore, about $57 \%$ are male, about $74 \%$ are white and approximately $33 \%$ of respondents have a household income of over
$\$ 100,000$. The majority of respondents are married $(57.3 \%)$ and have no children $(66.9 \%)$. Finally, about $35 \%$ are two-person households and approximately $75 \%$ had owned or bought their living quarters by themselves or someone else.

A breakdown of responses for public risk perceptions of other people's use of handguns is presented in Table 2. Using a scale of one to five, the results show that the mean public risk perception for other people's use of handguns is 3.6. If we consider rankings of 1 and 2 a low risk, 3 a moderate risk and 4 and 5 a high risk (3-point Likert scale), this finding suggests that the American public perceives other people using a handgun as highly risky. Using the same 3-point Likert scale above, about $19 \%$ of respondents report that other people's use of handguns is a low risk. In addition, approximately 27 and $55 \%$ report that other people's use of handguns is moderately risky and highly risky, respectively. Considering these findings together, we conclude that the American public perceives other people's use of a handgun to be very risky.

The results of the intercorrelations, which are presented in Table 3, show a positive and significant relationship between both age and education and risk perceptions of other people's use of handguns. In addition, the intercorrelations show that there is a negative and significant relationship between both male and whites and risk perceptions of other people's use of handguns.

Table 4 shows the results of the t-tests for gender and ethnicity. The results of these tests show that females have slightly higher risk perceptions of another person using a handgun (3.9) than males (3.4) and the difference between the two groups is statistically significant $(p<0.001)$. Table 4 also shows that minorities have slightly higher risk perceptions of other people using handguns (4.0) than whites (3.5) and the difference is also statistically significant $(\mathrm{p}<0.001)$.

Table 5 displays the results from the ordered logit regression model. The results of this model show that the same four independent variables that are statistically significant $(p<0.01)$ in the intercorrelations are also significant in the ordered logit regression model. Specifically, age and education are positively and significantly related to risk perceptions of other people's use of handguns. Conversely, white and male are negatively and significantly related to risk perceptions of other people's use of handguns. In addition, there is a positive, but insignificant relationship between parental status, marital status and renters and risk perceptions of other people's use of handguns. Furthermore, just as in the intercorrelations, there is an insignificant relationship between household income and risk perceptions of other people's use of handguns. 
Jenna Tyler and Abdul-Akeem Sadiq / Journal of Social Sciences 2017, 13 (3): 124.133 DOI: $10.3844 / j s s p .2017 .124 .133$

Table 1. Demographic characteristics of respondents

\begin{tabular}{|c|c|c|c|c|c|}
\hline Variable & Frequency & Percent & Variable & Frequency & Percent \\
\hline Age & & & Gender & & \\
\hline $18-29$ & 298 & 16.3 & Male & 1,035 & 56.5 \\
\hline $30-44$ & 574 & 31.3 & Female & 796 & 43.5 \\
\hline $45-59$ & 672 & 36.7 & & & \\
\hline $60+$ & 287 & 16.7 & & & \\
\hline Household Income & & & Ethnicity & & \\
\hline Less than $\$ 14,999$ & 76 & 4.2 & White, non-Hispanic & 1,353 & 73.9 \\
\hline$\$ 15,000-\$ 29,999$ & 152 & 8.3 & Black, non-Hispanic & 156 & 8.5 \\
\hline$\$ 30,000-\$ 49,999$ & 291 & 15.9 & Hispanic & 193 & 10.6 \\
\hline$\$ 50,000-\$ 74,999$ & 409 & 22.3 & Other, non-Hispanic & 83 & 4.5 \\
\hline$\$ 75,000-\$ 99,999$ & 304 & 16.6 & $2+$ races, non-Hispanic & 46 & 2.5 \\
\hline Over $\$ 100,000$ & & 599 & & 32.7 & \\
\hline Education & & & Household Size & & \\
\hline Less than high school & 63 & 3.5 & 1 & 344 & 18.8 \\
\hline High school graduate & 370 & 20.2 & 2 & 633 & 34.6 \\
\hline Some college & 368 & 20.1 & 3 & 358 & 19.5 \\
\hline Associate degree & 209 & 11.4 & 4 & 309 & 16.9 \\
\hline Bachelor's degree & 478 & 26.1 & 5 or more & 187 & 10.2 \\
\hline Master's degree & 237 & 12.9 & & & \\
\hline Professional/Doctorate & 106 & 5.8 & & & \\
\hline Marital Status & & & Ownership of Living $Q$ & & \\
\hline Married & 1,050 & 57.3 & Owned/bought by you & eone & \\
\hline Widowed & 32 & 1.8 & & 1,376 & 75.2 \\
\hline Divorced & 172 & 9.4 & Rented for cash & 429 & 23.4 \\
\hline Separated & 19 & 1.0 & Occupied without payr & cash rent & \\
\hline Never Married & 421 & 23.0 & & 26 & 1.4 \\
\hline Living with partner & 137 & 7.5 & & & \\
\hline \multicolumn{6}{|l|}{ Parental Status } \\
\hline One or more kids & 606 & 33.1 & & & \\
\hline No kid & 1,225 & 66.9 & & & \\
\hline
\end{tabular}

Table 2. Breakdown of responses for public risk perceptions of other people's use of handguns

\begin{tabular}{llll}
\hline Scale & Frequency & Percent & Mean $=3.6$ \\
\hline 1 (Not at all Risky) & 82 & 4.5 & Std. Dev. $=1.2$ \\
2 & 253 & 14.1 & \\
3 & 478 & 26.6 & \\
4 & 424 & 23.6 & \\
5 (Highly Risky) & 560 & 31.2 & \\
Total & 1,797 & 100 & \\
\hline
\end{tabular}

Table 3. Intercorrelations among all variables $(\mathrm{N}=1,670)$

\begin{tabular}{|c|c|c|c|c|c|c|c|c|c|c|}
\hline & 1 & 2 & 3 & 4 & 5 & 6 & 7 & 8 & 9 & 10 \\
\hline 1. Handguns & 1.00 & & & & & & & & & \\
\hline 2. Age & $0.08 * * *$ & 1.00 & & & & & & & & \\
\hline 3. Male & $-0.20 * * *$ & -0.02 & 1.00 & & & & & & & \\
\hline 4. Household Income & 0.00 & $0.09 * * *$ & $0.09 * * *$ & 1.00 & & & & & & \\
\hline 5. Education & $0.10 * * *$ & 0.02 & 0.01 & $0.37 * * *$ & 1.00 & & & & & \\
\hline 6. White & $-0.14 * * *$ & $0.10 * * *$ & -0.03 & $0.15 * * *$ & $0.14 * * *$ & 1.00 & & & & \\
\hline 7. Kids & -0.00 & $-0.21 * * *$ & $0.08 * * *$ & $0.09 * * *$ & -0.02 & -0.04 & 1.00 & & & \\
\hline 8. Household Size & -0.00 & $-0.21 * * *$ & $0.08 * * *$ & $0.22 * * *$ & $-0.05 * *$ & $-0.08 * * *$ & $0.69 * * *$ & 1.00 & & \\
\hline 9. Marital Status & 0.03 & $-0.35 * * *$ & $-0.13 * * *$ & $-0.28 * * *$ & $-0.04 *$ & $-0.14 * * *$ & $-0.23 * * *$ & $-0.26^{* * *}$ & 1.00 & \\
\hline 10. Rent & 0.01 & $-0.21 * * *$ & -0.01 & $-0.37 * * *$ & $-0.09 * * *$ & $-0.15 * * *$ & $-0.05 * *$ & $-0.14 * * *$ & $0.29 * * *$ & 1.00 \\
\hline
\end{tabular}

Note: ${ }^{*} \mathrm{p}<0.10 * * \mathrm{p}<0.05 * * * \mathrm{p}<0.01$ 
Table 4. T-test results for gender and ethnicity

\begin{tabular}{lllll}
\hline & $\mathrm{N}$ & Mean & Se & t-test \\
\hline Female & 781 & 3.90 & 0.04 & 0.04 \\
Male & 1016 & 3.42 & 0.06 & 8.75 \\
Difference & & 0.48 & 0.06 & $0.000 * * *$ \\
Minorities & 339 & 3.95 & 0.03 & 0.07 \\
White & 1335 & 3.52 & 0.43 & $0.00 * * *$ \\
Difference & & 0.41 & \\
\hline$* *$ p $<0.001$ & & &
\end{tabular}

Table 5. Ordered logit regression results

\begin{tabular}{lll}
\hline Variable & Coef. & Std. error \\
\hline Age & $0.27^{* * *}$ & 0.05 \\
Male & $-0.81^{* * *}$ & 0.09 \\
Household Income & -0.02 & 0.04 \\
Education & $0.14^{* * *}$ & 0.03 \\
White & $-0.81^{* * *}$ & 0.12 \\
Kids & 0.14 & 0.13 \\
Household Size & 0.03 & 0.05 \\
Marital Status & 0.04 & 0.03 \\
Rent & 0.05 & 0.11 \\
${ }^{* p}<0.10, * * \mathrm{p}<0.05$, & $\mathrm{N}=1,674$ & \\
$* * * \mathrm{p}<0.01$ & Pseudo $\mathrm{R}^{2}=0.03$ & \\
& Wald $x^{2}=166.05$ & \\
& Prob $x^{2}=0.0000$ & \\
\hline
\end{tabular}

Considering all of the analyses together, the results provide support for Hypotheses 1, 2, 3 and 4, but not Hypothesis 5. Specifically, the results from the intercorrelations and ordered logit regression model show that there is a positive and significant relationship between both age and education and risk perceptions of other people's use of handguns, thus supporting Hypotheses 1 and 2. Moreover, the results from the intercorrelations, ordered logit regression and t-tests provide support for Hypotheses 3 and 4, as they show a negative and significant relationship between both males and whites and public risk perceptions of other people's use of handguns. The findings, however, do not provide support for Hypothesis 5, as the results revealed an insignificant relationship between household income and risk perceptions of other people's use of handguns. In the following section, we provide explanations for these findings.

\section{Discussion}

The two objectives of this study are to understand public risk perceptions of other people's use of handguns and the influence of demographics on public risk perceptions of other people's use of handguns. With regard to the former, the results indicate that the public has a high level of risk perception of another person using a handgun. This finding corroborates other studies employing the psychometric paradigm that found the American public has a relatively high-risk perception of handguns (e.g., Cutter et al. 1992; Slovic et al. 1979). However, when we analyze risk perceptions of other people's use of handguns along various demographics and in the context of the cultural theory of risk, psychometric paradigm and official crime data, interesting insights emerge.

The results showed that age is positively associated with risk perceptions of other people's use of handguns. In other words, the older an individual is the higher their risk perception of another person using a handgun. Although this result supports those of previous risk perception studies (e.g., LaGrange and Ferraro, 1989; Kahan et al., 2007), this finding contradicts the psychometric paradigm and culture theory of risk. For example, because older individuals are significantly less likely to be a victim of firearm-related violence (Planty and Truman, 2013) and own firearms at a higher rate than younger individuals (NORC, 2015), theory would suggest that older individuals have a lower risk perception of another person using a handgun. Nonetheless, a plausible explanation for this finding is that the older an individual is, the more likely they are to learn about have experienced gun violence through the mass media (Wallace, 2015).

According to both the intercorrelations and the ordered logit regression, the higher the educational level, the higher the risk perceptions of another person using a handgun. This result is in line with those of Joslyn and Haider-Markel (2013; Kahan et al., 2007). One explanation for this result, according to Joslyn and Haider-Markel (2013), is that more educated individuals have the cognitive capacity to process complex information. In the context of this research, Joslyn and Haider-Markel's (2013) argument suggests that the more educated have the mental capacity to process and comprehend issues regarding the use of handguns and the consequences associated with using handguns. Another possible explanation for this result is that because individuals with a bachelor's degree or higher are less likely to own a firearm in comparison to those with a high school degree or less (Hepburn et al., 2007), more educated individuals may perceive the risk of other people's use of a handgun to be higher. To investigate this argument, we conducted an additional analysis by comparing the risk perceptions of other people's use of a handgun of individuals with less than a bachelor's degree and those with at least a bachelor's degree. The result indicates that the latter have a higher risk perception than the former (Table 6 in the appendix). 
Table 6. Sensitivity analysis: Ordered logit regression results

\begin{tabular}{lll}
\hline Variable & Coef. & Std. Error \\
\hline Age & $0.45^{* * *}$ & 0.13 \\
Male & $-0.83^{* * *}$ & 0.09 \\
Household Income & -0.06 & 0.10 \\
Education & $0.146^{* * *}$ & 0.09 \\
White & $-0.78^{* * *}$ & 0.12 \\
Kids & 0.02 & 0.13 \\
Household Size & 0.03 & 0.05 \\
Marital Status & 0.02 & 0.03 \\
Rent & 0.04 & 0.10 \\
$* \mathrm{p}<0.10, * * \mathrm{p}<0.05$, & $\mathrm{N}=1,674$ & \\
$* * * \mathrm{p}<0.01$ & Pseudo $\mathrm{R}^{2}=0.03$ & \\
& Wald $x^{2}=1546.56$ & \\
& Prob $x^{2}=0.0000$ & \\
\hline
\end{tabular}

The results of the intercorrelations, t-test and ordered logit regression show that men have significantly lower risk perceptions of other people's use of a handgun than women. This result corroborates an extensive list of studies showing lower risk perceptions of various hazardous activities and technologies by men in comparison to women (Barke et al., 1997; Gustafson, 1999; Kahan et al., 2007). For example, Kahan et al. (2007) found that women had a higher risk perception of the dangerousness of guns than men. The reasons why women tend to have higher risk perceptions of various hazardous activities and technologies than men have been the focus of previous research (e.g., Davidson and Freudenburg, 1996). Davidson and Freudenburg (1996) argue that the role of women in society such as a nurturer and caregiver makes them more concerned about the well-being of others than men. This argument offers a plausible explanation for our results; women being more concerned than men about the safety of others have relatively higher risk perceptions of another person using a handgun. Additionally, because women are more attuned to the possibility of being a victim of a violent attack (Kahan and Braman, 2003), it is not surprising that they have higher risk perceptions of another person using a handgun than men. Another possible explanation for this finding is that because men are more likely to own a gun in comparison to women (NORC, 2015), they will perceive the risk of handguns to be lower. This explanation aligns with the cultural theory of risk, which suggests that the more an individual believes the hazard supports their way of life, the lower they will perceive the risk of the hazard (Song, 2014).

With regards to ethnicity, the results of the inter correlations, t-test and ordered logit regression all show that whites have significantly lower risk perceptions of other people's use of handguns than blacks and Hispanics. This result is in line with previous studies that demonstrated minorities have higher risk perceptions of various hazardous activities and technologies than whites (Johnson, 2002; Lindell and Hwang, 2008). It is highly plausible that minorities maintain higher risk perceptions of other people's use of a handgun because they have directly experienced the risks associated with firearms at a higher rate than whites. For example, in 2010, the rate of firearm homicides for blacks was 14.6 per 100,000 , compared to 1.9 per 100,000 for whites in the United States (Planty and Truman, 2013). In addition, similar to education and gender, because minority groups are less likely to own a firearm (NORC, 2015), it makes sense that they have higher risk perceptions of other people's use of handguns.

\section{Conclusion}

Despite the ubiquitous gun control debate, which is sometimes amplified by prominent gun violence incidents and the extensive scholarship on crime and firearms, there is no systematic examination of public risk perceptions of other people's use of handguns. In addition, the relationship between public risk perceptions of other people's use of handguns and demographic characteristics such as age, educational level, gender, ethnicity and household income has yet to be studied. These two gaps are the motivation for this study. By addressing these lacunae, this study may be able to provide valuable information needed to design appropriate gun safety policies. For example, a policymaker equipped with information on the public's level of risk perception of other people using a handgun can compare this information with actuarial evidence of gun violence. Such a comparison may engender the need to develop risk communication programs and policies on gun safety to reconcile these differences. Our results regarding the second research question suggests that such reconciliation will need to consider differences in demographic characteristics.

A few study limitations are worth mentioning. First, there are other predictors of risk perception that were not controlled for in this study such as gun ownership (Joslyn and Haider-Markel, 2013), political party affiliation (Joslyn and Haider-Markel, 2013) and cultural worldviews (Kahan et al., 2007) because we did not collect information on these variables. Second, this study explores public risk perceptions of handguns using only one survey question and without any follow up questions. Third, this study did not measure under what conditions respondents perceived the risk of another person using a handgun or how respondents perceived risk in general. Fourth, this study is cross-sectional in nature. As such, we are unable to establish causality between risk perceptions of other people's use of handguns and the demographic characteristics of interest. Fifth, our study did not examine the reasons why different demographic groups have varying levels of risk perceptions of another person using a handgun. Sixth, the survey instrument did not gather information on the root causes of risk perceptions or on how risk 
perceptions vary between the use of another individual's handgun and an individual's own handgun. Seventh, this study did not explore the cognitive state of mind of individuals just prior to another person operating a handgun. Future studies on risk perceptions of firearms should seek to address these limitations.

Despite these limitations, our study provides a good starting point for understanding public risk perceptions of handguns, as well as the relationship between demographic characteristics and risk perceptions of handguns. More research in this area is urgently needed. First, future research should extend this study by including other relevant, but omitted predictors such as political party affiliation, gun ownership and cultural worldviews. Second, future studies should explicitly employ the psychometric paradigm and the cultural theory of risk to explore public risk perceptions of other people's use of handguns. Third, scholars should examine our research questions using panel data. Such a study may enable us to have a better understanding of the temporal changes or stability in public risk perceptions of other people's use of handguns as well as the relationship between public risk perceptions of other people's use of handguns and demographic characteristics over time. Finally, future research should examine the reasons why there are differences in public's risk perceptions of other people's use of handguns among various demographic groups.

\section{Funding Information}

The authors are grateful to the School of Public and Environmental Affairs for providing the funding for this study.

\section{Author Contributions}

Jenna Tyler assisted with developing the theoretical foundation, writing the manuscript and proof reading and editing. Abdul-Akeem Sadiq assisted with data collection, data analysis and proof reading and editing.

\section{Ethics}

The authors declare that they do not have any conflict of interest.

\section{References}

AAPOR, 2015. Standard definitions. American Association for Public Opinion Research.

Barke, R.P., H. Jenkins-Smith and P. Slovic, 1997. Risk perceptions of men and women scientists. Soc. Sci. Q., 78: 167-176.

Boholm, A., 1998. Comparative studies of risk perception: A review of twenty years of research. J. Risk Res., 1: 135-163. DOI: 10.1080/136698798377231
Celinska, K., 2007. Individualism and collectivism in America: The case of gun ownership and attitudes toward gun control. Sociol. Perspectives, 50: 229-247. DOI: 10.1525/sop.2007.50.2.229

Chiricos, T., R. McEntire and M. Gertz, 2000. Perceived racial and ethnic composition of neighborhood and perceived risk of crime. Soc. Problems, 48: 322-340. DOI: $10.1525 / \mathrm{sp} .2001 .48 .3 .322$

Cohn, D., P. Taylor, M.H. Lopez, C.A. Gallagher and K. Parker et al., 2013. Gun homicide rate down 49\% since 1993 peak; Public Unaware. Pew Research Center.

Cutter, S.L., J. Tiefenbacher and W.D. Solecki, 1992. En-gendered fears: Femininity and technological risk perception. Organiz. Environ., 6: 5-22. DOI: $10.1177 / 108602669200600102$

Davidson, D.J. and W.R. Freudenburg, 1996. Gender and environmental risk concerns: A review and analysis of available research. Environ. Behav., 28: 302-339. DOI: 10.1177/0013916596283003

Dosman, D.M., W.L. Adamowicz and S.E. Hrudey, 2001. Socioeconomic determinants of health- and food safety-related risk perceptions. Risk Anal., 21: 307-318. DOI: 10.1111/0272-4332.212113

Douglas, M. and A. Wildavsky, 1983. Risk and Culture: An Essay on the Selection of Technological and Environmental Dangers. 1st Edn., University of California Press, Berkeley, CA., ISBN-10: 0520907396, pp: 224.

Finucane, M.L., P. Slovic, C.K. Mertz, J. Flynn and T.A. Satterfield, 2000. Gender, race and perceived risk: The 'white male' effect. Health Risk Society, 2: 159-172. DOI: 10.1080/713670162

Fischhoff, B., P. Slovic, S. Lichtenstein, S. Read and B. Combs, 1978. How safe is safe enough? A psychometric study of attitudes towards technological risks and benefits. Policy Sci., 9: 127-152. DOI: 10.1007/BF00143739

Grassel, K.M., G.J. Wintemute, M.A. Wright and M.P. Romero. 2003. Association between handgun purchase and mortality from firearm injury. Injury Prevent., 9: 48-52. DOI: 10.1136/ip.9.1.48

Grothmann, T. and F. Reusswig, 2006. People at Risk of flooding: Why some residents take precautionary action while others do not. Nat. Hazards, 38: 101-120. DOI: 10.1007/s11069-005-8604-6

Gustafson, P.E., 1999. Gender differences in risk perception: Theoretical and methodological erspectives. Risk Anal., 18: 805-811. DOI: $10.1111 / \mathrm{j} .1539-6924.1998 . t b 01123 . x$

Hemenway, D., D. Azrael and M. Miller, 2000. Gun use in the United States: Results from two national surveys. Injury Prevent., 6: 263-267. DOI: 10.1136/ip.6.4.263 
Hepburn, L., M. Miller, D. Azrael and D. Hemenway, 2007. The US gun stock: Results from the 2004 national firearms survey. Injury Prevent., 13: 15-19. DOI: 10.1136/ip.2006.013607

Hraba, J., W. Bao, F.O. Lorenz and Z. Pechačová, 1998. Perceived risk of crime in the Czech Republic. J. Res. Crime Delinquency, 35: 225-242. DOI: $10.1177 / 0022427898035002005$

Johnson, B.B., 2002. Gender and race in beliefs about outdoor air pollution. Risk Anal., 22: 725-738. DOI: 10.1111/0272-4332.00064

Joslyn, M.R. and D.P. Haider-Markel, 2013. The politics of causes: Mass shootings and the cases of the Virginia Tech and Tucson tragedies. Soc. Sci. Q., 94: 410-423. DOI: $10.1111 / \mathrm{j} .1540-6237.2012 .00894 . x$

Kahan, D.M. and D. Braman, 2003. More statistics, less persuasion: A cultural theory of gun-risk perceptions. Univ. Pennsylvania Law Rev., 151: 1291-1327. DOI: $10.2307 / 3312930$

Kahan, D.M., D. Braman, J. Gastil, P. Slovic and C.K. Mertz, 2007. Culture and identity-protective cognition: Explaining the white-male effect in risk perception. J. Empirical Legal Stud., 4: 465-505. DOI: $10.1111 / \mathrm{j} .1740-1461.2007 .00097 . x$

Kahan, D.M., H. Jenkins-Smith and D. Braman, 2011. Cultural cognition of scientific consensus. J. Risk Res., 14: 147-174.

DOI: $10.1080 / 13669877.2010 .511246$

Kalesan, B., M.D. Villarreal, K.M. Keyes and S. Galea, 2016. Gun ownership and social gun culture. Injury Prevent., 22: 216-220.

DOI: 10.1136/injuryprev-2015-041586

Konty, M. and B. Schaefer, 2012. Small arms mortality: Access to firearms and lethal violence. Soc. Spectrum, 32: 475-490.

DOI: $10.1080 / 02732173.2012 .700832$

LaGrange, R.L. and K.F. Ferraro, 1989. Assessing age and gender differences in perceived risk and fear of crime. Criminology., 27: 697-720.

DOI: $10.1111 / \mathrm{j} .1745-9125.1989 . t b 01051 . x$

Lindell, M.K. and S.N. Hwang, 2008. Households' perceived personal risk and responses in a multihazard environment. Risk Anal., 28: 539-556. DOI: $10.1111 /$ j.1539-6924.2008.01032.x

Mesch, G.S., 2000. Perceptions of risk, lifestyle activities and fear of crime. Deviant Behav., 21 : 47-62. DOI: 10.1080/016396200266379

NORC, 2015. Trends in gun ownership in the United States, 1972-2014. National Opinion Research Center.

Planty, M. and J.L. Truman, 2013. Firearm violence, 1993-2011.

Rader, N.E., D.C. May and S. Goodrum, 2007. An Empirical assessment of the "threat of victimization:" Considering fear of crime, perceived risk, avoidance and defensive behaviors. Soc. Spectrum, 27: 475-505. DOI: 10.1080/02732170701434591
Rountree, P.W. and K.C. Land, 1996. Perceived risk versus fear of crime: Empirical evidence of conceptually distinct reactions in survey data. Soc. Forces, 74: 1353-1376. DOI: 10.2307/2580354

Sadiq, A.A. and J.D. Graham, 2016. Exploring the predictors of organizational preparedness for natural disasters. Risk Anal., 36: 1040-1053. DOI: $10.1111 /$ risa. 12478

Sadiq, A.A., K. Tharp, J.D. Graham and J. Tyler, in press. Temporal stability and changes in risk perception rankings of hazardous activities and technologies. J Risk Res.

Savage, I., 1993. Demographic influences on risk perceptions. Risk Anal., 13: 413-420. DOI: $10.1111 / \mathrm{j} .1539-6924.1993 . t b 00741 . x$

Sjöberg, L., B.E. Moen and T. Rundmo, 2004. Explaining Risk Perception: An Evaluation of the Psychometric Paradigm in Risk Perception Research. 1st Edn., Rotunde, Trondheim, Norway, ISBN-10: 82-7892-024-9.

Slovic, P., B. Fischhoff and S. Lichtenstein, 1979. Rating the risks. Environ. Sci. Policy Sustainable Dev., 21: 14-39. DOI: $10.1080 / 00139157.1979 .9933091$

Song, G., 2014. Understanding public perceptions of benefits and risks of childhood vaccinations in the USA. Risk Anal., 34: 541-55. DOI: 10.1111/risa.12114

Stroebe, W., 2015. Firearm availability and violent death: The need for a culture change in attitudes toward guns. Anal. Soc. Issues Public Policy, 16: 7-35. DOI: 10.1111/asap. 12100

Teret, S.P., D.W. Webster, J.S. Vernick, T.W. Smith and D. Leff et al., 1998. Support for new policies to regulate firearms-Results of two national surveys. New Engl. J. Med., 339: 813-818. DOI: 10.1056/NEJM199809173391206

Wallace, L.N., 2015. Responding to violence with guns: Mass shootings and gun acquisition. Soc. Sci. J., 52: 156-167. DOI: 10.1016/j.soscij.2015.03.002

Wilcox, P., N. Quisenberry and S. Jones, 2003. The built environment and community crime risk interpretation. J Res Crime Delinq., 40: 322-345. DOI: $10.1177 / 0022427803253801$

Webster, D.W., J.S. Vernick and L.M. Hepburn, 2001. Relationship between licensing, registration and other gun sales laws and the source state of crime guns. Injury Prevent., 7: 184-189. DOI: 10.1136/ip.7.3.184

White, C.M., M. Gummerum and Y. Hanoch, 2015. Adolescents' and young adults' online risk taking: The role of gist and verbatim representations. Risk Anal., 35: 1407-1422. DOI: 10.1111/risa.12369 